\title{
Permanence of moment estimates for $p$-products of convex bodies
}

\author{
by \\ Ulrich Brehm, Hendrik Vogt, and \\ Jürgen Voigt (Dresden)
}

\begin{abstract}
It is shown that two inequalities concerning second and fourth moments of isotropic normalized convex bodies in $\mathbb{R}^{n}$ are permanent under forming $p$-products. These inequalities are connected with a concentration of mass property as well as with a central limit property. An essential tool are certain monotonicity properties of the $\Gamma$-function.
\end{abstract}

Introduction. The topic of the present paper originated from and is connected with a version of the central limit theorem in the context of convex bodies in geometry.

A normalized convex body $K \subseteq \mathbb{R}^{n}$ is a convex compact set of volume 1 whose centre of mass is at the origin. A normalized convex body is isotropic if its ellipsoid of inertia is a Euclidean ball, i.e., if

$$
L_{K}^{2}:=\int_{K}(x \cdot u)^{2} d x
$$

is independent of $u \in S^{n-1}$, the unit sphere of $\mathbb{R}^{n}$.

The main objective of the present paper is the validity of two inequalities concerning the moments

$$
M_{2, n}(K):=\int_{K}|x|^{2} d x \quad\left(=n L_{K}^{2}\right), \quad M_{4, n}(K):=\int_{K}|x|^{4} d x
$$

of isotropic normalized convex bodies $K \subseteq \mathbb{R}^{n}$. Here and in the following, the norm $|\cdot|$ always denotes the Euclidean norm. In [9] it has been shown that the inequalities

2000 Mathematics Subject Classification: Primary 52A20; Secondary 33B15, 60F25.

Key words and phrases: convex body, isotropic, moment inequalities, Gamma function, central limit theorem.

Research of H. Vogt supported by the DFG.

Research of J. Voigt partly supported by the DFG. 


$$
\begin{aligned}
M_{2, n}(K) & \leqslant M_{2, n}\left(\Delta_{n}\right)=\frac{n}{(n+1)(n+2)} \cdot \frac{n !^{2 / n}}{(n+1)^{1 / n}} \\
\frac{M_{4, n}(K)}{M_{2, n}(K)^{2}} & \leqslant \frac{M_{4, n}\left(\Delta_{n}\right)}{M_{2, n}\left(\Delta_{n}\right)^{2}}=\frac{(n+1)(n+2)}{(n+3)(n+4)}\left(1+\frac{2}{n}+\frac{6}{n+1}\right)
\end{aligned}
$$

are valid for isotropic normalized Euclidean balls, cubes, and cross polytopes, where $\Delta_{n}$ denotes the normalized regular simplex in $\mathbb{R}^{n}$. Moreover, it is shown that these inequalities persist under the operations of forming isotropic normalized cones, cartesian products, and joins. Here we extend these results and show that $(0.1),(0.2)$ also persist under forming $p$-products of isotropic normalized convex bodies. This implies, in particular, that (0.1), $(0.2)$ are valid for $p$-balls in $\mathbb{R}^{n}\left(\ell_{p}^{n}\right.$-balls $)$.

In order to motivate these investigations we recall the notion of central limit property for isotropic normalized convex bodies. We regard an isotropic normalized convex body $K \subseteq \mathbb{R}^{n}$, with the Lebesgue measure $\lambda_{n}$, as a probability space. For a unit vector $u \in S^{n-1}$ we define a random variable $X_{K, u}: K \rightarrow \mathbb{R}$ by

$$
X_{K, u}(x):=x \cdot u \text {. }
$$

The density of its distribution is given by

$$
\varphi_{K, u}(t)=\lambda_{n-1}(\{x \in K: x \cdot u=t\}) \quad(t \in \mathbb{R}) .
$$

The central limit property of a set of isotropic normalized convex bodies roughly means that the distributions of $X_{K, u}$ are close to Gaussian distributions for large $n$ and "most" directions $u \in S^{n-1}$.

In order to make this more precise we introduce the set $\mathcal{K}_{n}$ of all isotropic normalized convex bodies in $\mathbb{R}^{n}$, and we define

$$
\mathcal{K}:=\bigcup_{n=1}^{\infty} \mathcal{K}_{n} .
$$

Following [3], [2], we say that a set $\mathcal{M} \subseteq \mathcal{K}$ has the central limit property if

$$
\begin{aligned}
\sup _{K \in \mathcal{K}_{n} \cap \mathcal{M}} \mu_{n-1}\left(\left\{u \in S^{n-1}:\left\|\varphi_{K, u}-g_{L_{K}^{2}}\right\|_{1}>\varepsilon\right\}\right) & \rightarrow 0 \\
\text { as } n & \rightarrow \infty, \text { for all } \varepsilon>0 .
\end{aligned}
$$

Here,

$$
g_{\sigma^{2}}(t)=\frac{1}{\sqrt{2 \pi \sigma^{2}}} \exp \left(-\frac{t^{2}}{2 \sigma^{2}}\right)
$$

denotes the Gaussian density, and $\mu_{n-1}$ the surface measure on $S^{n-1}$, normalized to be a probability measure. In [2] it is shown that the central limit property can be expressed equivalently using distribution functions or the $L_{\infty}$-norm instead of the $L_{1}$-norm (the latter under an additional assumption). In [3], the central limit property is shown for the set of cubes and 
Euclidean balls. In [1] it is shown that, for symmetric isotropic normalized convex bodies, the central limit property (in terms of distribution functions) can be derived from a "concentration hypothesis". In a more general context, it is shown in [10] that a concentration of mass property implies a central limit property. This concentration of mass property (or concentration hypothesis) means that, for high dimensions, the volume of the bodies under consideration is mainly concentrated in a certain spherical shell. Using [10], and assuming the concentration of mass property for a set $\mathcal{M} \subseteq \mathcal{K}$, it is shown in [2] that $\mathcal{M}$ has the central limit property formulated above.

The role of inequalities $(0.1),(0.2)$ in this context is that they imply the concentration of mass property; cf. [9]. As a consequence, the subset $\mathcal{T}$ of all bodies $K$ in $\mathcal{K}$ satisfying these inequalities has the central limit property. An inequality similar to (but weaker than) (0.2) for $\ell_{p}^{n}$-balls was shown in [1] in order to conclude the concentration hypothesis in this case. The desire to prove $(0.1),(0.2)$ for $\ell_{p}^{n}$-balls was the starting point of the present paper. We refer to [4] for a study of the asymptotic behaviour, as $n \rightarrow \infty$, of the volume of sections of $\ell_{p}^{n}$-balls $(0<p \leqslant \infty)$.

As a side remark we mention that for bodies $K \in \mathcal{K}$ satisfying (0.1) one obtains $L_{K} \leqslant 1 / e$. It is an open question whether there is a universal bound on $L_{K}$ for $K \in \mathcal{K}$ (see [6, Sec. 5]). As a second remark we mention that the bound in (0.2) converges to 1 as $n \rightarrow \infty$, which is the essential feature for deriving the concentration of mass property (cf. [9, Sec. 1]).

In Section 1 we define the (isotropic normalized) p-product of isotropic normalized convex bodies, and we compute the moments in terms of the moments of the given bodies.

In Section 2 we show that inequalities (0.1) and (0.2) are permanent under forming $p$-products.

In Section 3 we give more precise information on the $p$-balls in $\mathbb{R}^{n}$.

The most sophisticated material of this paper, in our opinion, is contained in Section 4. Having derived the expressions (1.5) and (1.8), it was not obvious how to handle the abundance of $\Gamma$-functions occurring in these expressions. The technical tools concerning the $\Gamma$-function presented in this section are essential for Sections 2 and 3 and should also be of independent interest.

Finally, in Section 5 we show that the expression $M_{4, n}(K) / M_{2, n}(K)^{2}$ considered in (0.2) takes its minimal value for the Euclidean ball. The corresponding property for $M_{2, n}(K)$ is immediate.

1. The $p$-product of convex bodies and its moments. For $j=$ 1,2 , we assume $n_{j} \in \mathbb{N}$, and we take an isotropic normalized convex body $K_{j} \subseteq \mathbb{R}^{n_{j}}$. Let $|\cdot|_{j}$ denote the gauge functional of $K_{j}$. (In general, $|\cdot|_{j}$ will 


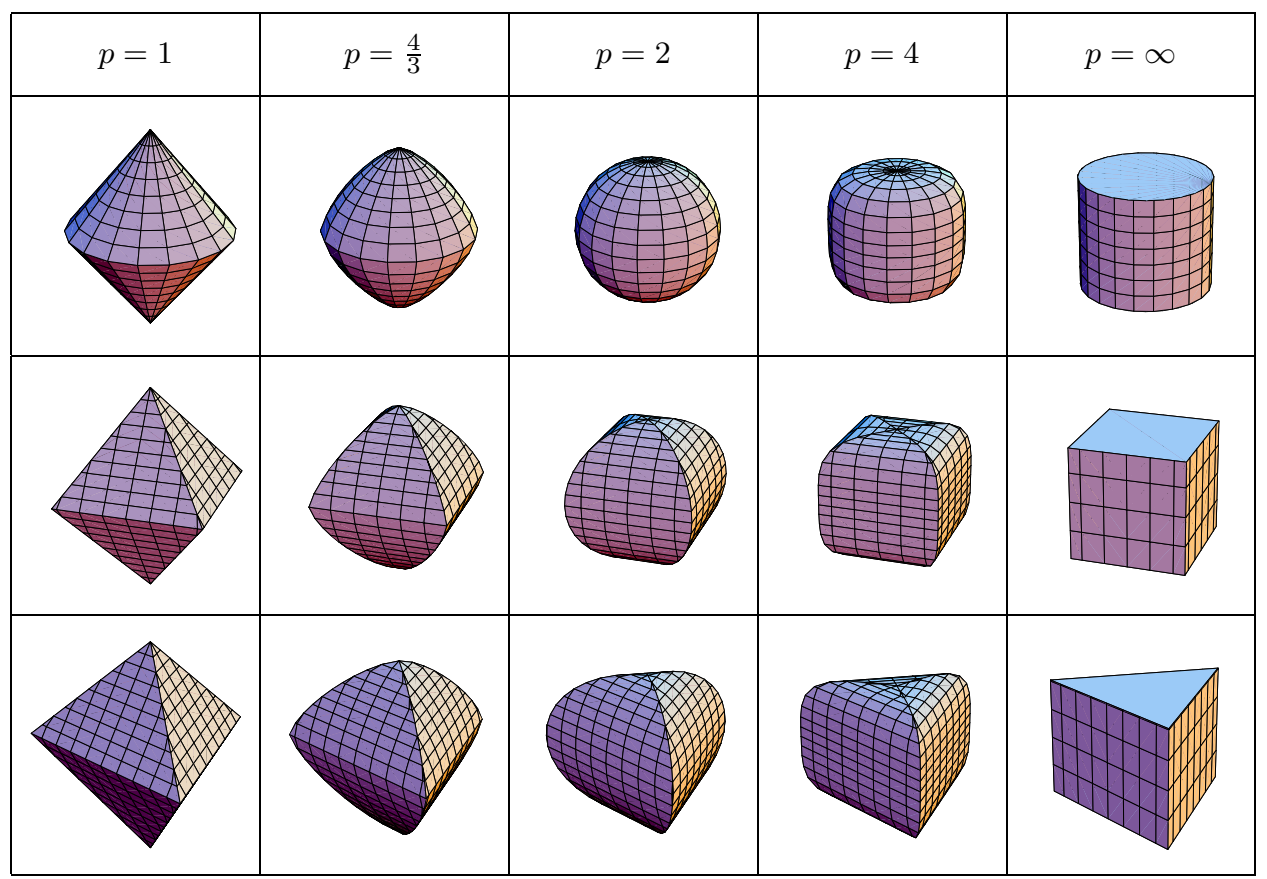

Fig. 1. Examples of $p$-products

be sublinear; it will be a norm if and only if $K_{j}$ is symmetric.) Moreover, define $n:=n_{1}+n_{2}$, and let $1 \leqslant p \leqslant \infty$. On $\mathbb{R}^{n}$ we define the sublinear functional

$$
\left|\left(x_{1}, x_{2}\right)\right|_{p}:= \begin{cases}\left(\left|x_{1}\right|_{1}^{p}+\left|x_{2}\right|_{2}^{p}\right)^{1 / p} & \text { for } 1 \leqslant p<\infty \\ \max \left(\left|x_{1}\right|_{1},\left|x_{2}\right|_{2}\right) & \text { for } p=\infty\end{cases}
$$

where $x_{j} \in \mathbb{R}^{n_{j}}(j=1,2)$. We define the $p$-product $K_{1} \times{ }_{p} K_{2}$ (which is not a normalized convex body) by

$$
K_{1} \times_{p} K_{2}:=\left\{\left(x_{1}, x_{2}\right) \in \mathbb{R}^{n}:\left|\left(x_{1}, x_{2}\right)\right|_{p} \leqslant 1\right\} .
$$

We note that $K_{1} \times_{\infty} K_{2}$ is just the ordinary cartesian product, whereas

$$
K_{1} \times_{1} K_{2}=\left\{\left(t x_{1},(1-t) x_{2}\right): x_{j} \in K_{j}(j=1,2), 0 \leqslant t \leqslant 1\right\}
$$

is the join of $K_{1}$ and $K_{2}$. These cases were studied in [9].

Note that $\int_{K_{1} \times_{p} K_{2}}(x \cdot u)(x \cdot v) d x=0$ if $u \in \mathbb{R}^{n_{1}} \times\{0\}, v \in\{0\} \times \mathbb{R}^{n_{2}}$, because the centre of mass of $K_{1}$ and $K_{2}$, respectively, is the origin. Moreover, $\int_{K_{1} \times_{p} K_{2}}(x \cdot u)^{2} d x$ is constant on $S^{n_{1}-1} \times\{0\}$ and on $\{0\} \times S^{n_{2}-1}$ because $K_{1}$ and $K_{2}$ are isotropic. Therefore, the isotropic normalized body associated with $K_{1} \times_{p} K_{2}$ is of the form $\left(\alpha_{1} K_{1}\right) \times_{p}\left(\alpha_{2} K_{2}\right)$, where $\alpha_{1}, \alpha_{2}$ are suitable scaling factors. In Figure 1 we show some examples for the case $n_{1}=2, n_{2}=1$, with different $p$, where $K_{1}$ is a circle, a square and an equilateral triangle, respectively (and $K_{2}=[-1 / 2,1 / 2]$ ). 
In order to find the correct scaling factors we first compute the volume of $K_{1} \times{ }_{p} K_{2}$. The method we use for doing so is inspired by the computation of the volume of $p$-balls given in [7, p. 11].

First we note

$$
\int_{\mathbb{R}^{n}} e^{-\left|\left(x_{1}, x_{2}\right)\right|_{p}^{p}} d x=\int_{\mathbb{R}^{n_{1}}} e^{-\left|x_{1}\right|_{1}^{p}} d x_{1} \int_{\mathbb{R}^{n_{2}}} e^{-\left|x_{2}\right|_{2}^{p}} d x_{2} .
$$

Now,

$$
\begin{aligned}
\int_{\mathbb{R}^{n_{1}}} e^{-\left|x_{1}\right|_{1}^{p}} d x_{1} & =\int_{\mathbb{R}^{n_{1}}}\left(-\int_{\left|x_{1}\right|_{1}^{p}}^{\infty} \frac{d}{d t} e^{-t} d t\right) d x_{1} \\
& =\int_{0}^{\infty} \int_{\left|x_{1}\right|_{1}^{p} \leqslant t} d x_{1} e^{-t} d t=\int_{0}^{\infty} t^{n_{1} / p} e^{-t} d t V_{1}=\Gamma\left(n_{1} / p+1\right),
\end{aligned}
$$

with $V_{1}:=\operatorname{vol}_{n_{1}}\left(K_{1}\right)=1$. Computing the other integrals in (1.1) in the same way, we obtain

$$
\Gamma(n / p+1) \operatorname{vol}_{n}\left(\left(\alpha_{1} K_{1}\right) \times_{p}\left(\alpha_{2} K_{2}\right)\right)=\alpha_{1}^{n_{1}} \Gamma\left(n_{1} / p+1\right) \alpha_{2}^{n_{2}} \Gamma\left(n_{2} / p+1\right),
$$

and therefore the first requirement for the scaling factors $\alpha_{1}, \alpha_{2}>0$ amounts to

$$
\alpha_{1}^{n_{1}} \alpha_{2}^{n_{2}}=\frac{\Gamma(n / p+1)}{\Gamma\left(n_{1} / p+1\right) \Gamma\left(n_{2} / p+1\right)} .
$$

In order to obtain isotropy we compute second moments (recall that $|\cdot|$ denotes the Euclidean norm):

$$
\begin{aligned}
\int_{\mathbb{R}^{n}}\left|x_{1}\right|^{2} e^{-\left|\left(x_{1}, x_{2}\right)\right|_{p}^{p}} d x & =\int_{\mathbb{R}^{n_{1}}}\left|x_{1}\right|^{2}\left(-\int_{\left|x_{1}\right|_{1}^{p}}^{\infty} \frac{d}{d t} e^{-t} d t\right) d x_{1} \int_{\mathbb{R}^{n_{2}}} e^{-\left|x_{2}\right|_{2}^{p}} d x_{2} \\
& =\int_{0}^{\infty} \int_{\left|x_{1}\right|_{1}^{p} \leqslant t}\left|x_{1}\right|^{2} d x_{1} e^{-t} d t \Gamma\left(n_{2} / p+1\right) \\
& =\int_{0}^{\infty} t^{\left(n_{1}+2\right) / p} M_{2,1} e^{-t} d t \Gamma\left(n_{2} / p+1\right) \\
& =\Gamma\left(\left(n_{1}+2\right) / p+1\right) \Gamma\left(n_{2} / p+1\right) M_{2,1},
\end{aligned}
$$

where, for abbreviation, $M_{2, j}:=M_{2, n_{j}}\left(K_{j}\right)(j=1,2)$. The first expression in the last chain of equalities can also be computed as

$$
\begin{aligned}
\int\left|x_{1}\right|^{2} e^{-\left|\left(x_{1}, x_{2}\right)\right|_{p}^{p}} d x & =\int\left|x_{1}\right|^{2}\left(-\int_{|x|_{p}^{p}}^{\infty} \frac{d}{d t} e^{-t} d t\right) d x \\
& =\int_{0}^{\infty} \int_{|x|_{p}^{p} \leqslant t}\left|x_{1}\right|^{2} d x e^{-t} d t
\end{aligned}
$$




$$
\begin{aligned}
& =\int_{0}^{\infty} t^{(n+2) / p} e^{-t} d t \int_{K_{1} \times{ }_{p} K_{2}}\left|x_{1}\right|^{2} d x \\
& =\Gamma\left(\left(n_{1}+2\right) / p+1\right) \int_{K_{1} \times{ }_{p} K_{2}}\left|x_{1}\right|^{2} d x
\end{aligned}
$$

and therefore

$$
\int_{\left(\alpha_{1} K_{1}\right) \times_{p}\left(\alpha_{2} K_{2}\right)}\left|x_{1}\right|^{2} d x=\alpha_{1}^{n_{1}+2} \alpha_{2}^{n_{2}} \frac{\Gamma\left(\left(n_{1}+2\right) / p+1\right) \Gamma\left(n_{2} / p+1\right)}{\Gamma\left(\left(n_{1}+2\right) / p+1\right)} M_{2,1} .
$$

Now, isotropy requires

$$
\frac{1}{n_{1}} \int_{\left(\alpha_{1} K_{1}\right) \times_{p}\left(\alpha_{2} K_{2}\right)}\left|x_{1}\right|^{2} d x=\frac{1}{n_{2}} \int_{\left(\alpha_{1} K_{1}\right) \times_{p}\left(\alpha_{2} K_{2}\right)}\left|x_{2}\right|^{2} d x,
$$

which can be expressed as

$$
\begin{aligned}
\alpha_{1}^{2} \Gamma\left(\left(n_{1}+2\right) / p+1\right) \Gamma( & \left.n_{2} / p+1\right) \frac{M_{2,1}}{n_{1}} \\
& =\alpha_{2}^{2} \Gamma\left(n_{1} / p+1\right) \Gamma\left(\left(n_{2}+2\right) / p+1\right) \frac{M_{2,2}}{n_{2}} .
\end{aligned}
$$

With $\alpha_{1}, \alpha_{2}$ obtained from (1.2), (1.4) we define

$$
K_{1} *_{p} K_{2}:=\left(\alpha_{1} K_{1}\right) \times_{p}\left(\alpha_{2} K_{2}\right)
$$

as the isotropic normalized p-product of $K_{1}$ and $K_{2}$; we obtain

$$
\begin{aligned}
M_{2, n}\left(K_{1} *_{p} K_{2}\right) & n\left(\frac{1}{n_{1}} \int_{K_{1} *_{p} K_{2}}\left|x_{1}\right|^{2} d x\right)^{n_{1} / n}\left(\frac{1}{n_{2}} \int_{K_{1} *_{p} K_{2}}\left|x_{2}\right|^{2} d x\right)^{n_{2} / n} \\
= & n\left(\alpha_{1}^{n_{1}+2} \alpha_{2}^{n_{2}} \frac{\Gamma\left(\left(n_{1}+2\right) / p+1\right) \Gamma\left(n_{2} / p+1\right)}{\Gamma((n+2) / p+1)} \cdot \frac{M_{2,1}}{n_{1}}\right)^{n_{1} / n} \\
& \times\left(\alpha_{1}^{n_{1}} \alpha_{2}^{n_{2}+2} \frac{\Gamma\left(n_{1} / p+1\right) \Gamma\left(\left(n_{2}+2\right) / p+1\right)}{\Gamma((n+2) / p+1)} \cdot \frac{M_{2,2}}{n_{2}}\right)^{n_{2} / n} \\
= & n\left[\frac { ( \alpha _ { 1 } ^ { n _ { 1 } } \alpha _ { 2 } ^ { n _ { 2 } } ) ^ { n + 2 } } { \Gamma ( ( n + 2 ) / p + 1 ) ^ { n } } \left(\Gamma\left(\frac{n_{1}+2}{p}+1\right) \Gamma\left(\frac{n_{2}}{p}+1\right)^{\left.\frac{M_{2,1}}{n_{1}}\right)^{n_{1}}}\right.\right. \\
& \left.\times\left(\Gamma\left(\frac{n_{1}}{p}+1\right) \Gamma\left(\frac{n_{2}+2}{p}+1\right) \frac{M_{2,2}}{n_{2}}\right)^{n_{2}}\right]^{1 / n} \cdot
\end{aligned}
$$

By (1.2), this can be transformed into 


$$
\begin{aligned}
& M_{2, n}\left(K_{1} *_{p} K_{2}\right) \\
& \quad=n\left(\frac{f\left(n_{1}, 1 / p\right) f\left(n_{2}, 1 / p\right)}{f(n, 1 / p)}\left(\frac{M_{2, n_{1}}\left(K_{1}\right)}{n_{1}}\right)^{n_{1}}\left(\frac{M_{2, n_{2}}\left(K_{2}\right)}{n_{2}}\right)^{n_{2}}\right)^{1 / n},
\end{aligned}
$$

with the function

$$
f(n, \alpha):=\frac{\Gamma(\alpha(n+2)+1)^{n}}{\Gamma(\alpha n+1)^{n+2}} .
$$

The derivation of this formula is only valid for $p<\infty$, but the final expression is also true for $p=\infty$ (cartesian product); cf. [9, Sec. 3].

In order to compute $\left(M_{4, n} / M_{2, n}^{2}\right)\left(K_{1} *_{p} K_{2}\right)$ we note

$$
\int_{K_{1} \times{ }_{p} K_{2}}|x|^{4} d x=\int_{K_{1} \times{ }_{p} K_{2}}\left(\left|x_{1}\right|^{4}+2\left|x_{1}\right|^{2}\left|x_{2}\right|^{2}+\left|x_{2}\right|^{4}\right) d x .
$$

In the same way as above we compute

$$
\begin{aligned}
& \int_{K_{1} \times K_{p} K_{2}}\left|x_{1}\right|^{4} d x=\frac{\Gamma\left(\left(n_{1}+4\right) / p+1\right) \Gamma\left(n_{2} / p+1\right)}{\Gamma((n+4) / p+1)} M_{4,1}, \\
& \int_{K_{1} \times p K_{2}}\left|x_{1}\right|^{2}\left|x_{2}\right|^{2} d x=\frac{\Gamma\left(\left(n_{1}+2\right) / p+1\right) \Gamma\left(\left(n_{2}+2\right) / p+1\right)}{\Gamma((n+4) / p+1)} M_{2,1} M_{2,2} .
\end{aligned}
$$

In the following computations we use the fact that $M_{2}:=M_{2, n}\left(K_{1} *_{p} K_{2}\right)$ can be expressed by means of (1.3) as well as the symmetric expression. Thus,

$$
\begin{aligned}
& \frac{\int_{K_{1} *_{p} K_{2}}\left|x_{1}\right|^{4} d x}{M_{2}^{2}} \\
& =\frac{\alpha_{1}^{n_{1}+4} \alpha_{2}^{n_{2}} \frac{\Gamma\left(\left(n_{1}+4\right) / p+1\right) \Gamma\left(n_{2} / p+1\right)}{\Gamma((n+4) / p+1)} M_{4,1}}{\frac{n^{2}}{n_{1}^{2}} \alpha_{1}^{2\left(n_{1}+2\right)} \alpha_{2}^{2 n_{2}} \frac{\Gamma\left(\left(n_{1}+2\right) / p+1\right)^{2} \Gamma\left(n_{2} / p+1\right)^{2}}{\Gamma((n+2) / p+1)^{2}} M_{2,1}^{2}} \\
& =\frac{n_{1}^{2}}{n^{2}} \cdot \frac{1}{\alpha_{1}^{n_{1}} \alpha_{2}^{n_{2}}} \cdot \frac{\Gamma((n+2) / p+1)^{2}}{\Gamma((n+4) / p+1)} \cdot \frac{\Gamma\left(\left(n_{1}+4\right) / p+1\right)}{\Gamma\left(\left(n_{1}+2\right) / p+1\right)^{2}} \cdot \frac{1}{\Gamma\left(n_{2} / p+1\right)} \cdot \frac{M_{4,1}}{M_{2,1}^{2}} \\
& =\frac{n_{1}^{2}}{n^{2}} \cdot \frac{g\left(n_{1}, 1 / p\right)}{g(n, 1 / p)} \cdot \frac{M_{4,1}}{M_{2,1}^{2}},
\end{aligned}
$$

with the function

$$
g(n, \alpha):=\frac{\Gamma(\alpha n+1) \Gamma(\alpha(n+4)+1)}{\Gamma(\alpha(n+2)+1)^{2}}
$$

and 


$$
\begin{aligned}
& \frac{\int_{K_{1} *_{p} K_{2}}\left|x_{1}\right|^{2}\left|x_{2}\right|^{2} d x}{M_{2}^{2}} \\
& =\frac{\alpha_{1}^{n_{1}+2} \alpha_{2}^{n_{2}+2} \frac{\Gamma\left(\frac{n_{1}+2}{p}+1\right) \Gamma\left(\frac{n_{2}+2}{p}+1\right)}{\Gamma\left(\frac{n+4}{p}+1\right)} M_{2,1} M_{2,2}}{\frac{n}{n_{1}} \alpha_{1}^{n_{1}+2} \alpha_{2}^{n_{2}} \frac{\Gamma\left(\frac{n_{1}+2}{p}+1\right) \Gamma\left(\frac{n_{2}}{p}+1\right)}{\Gamma\left(\frac{n+2}{p}+1\right)} M_{2,1} \frac{n}{n_{2}} \alpha_{1}^{n_{1}} \alpha_{2}^{n_{2}+2} \frac{\Gamma\left(\frac{n_{1}}{p}+1\right) \Gamma\left(\frac{n_{2}+2}{p}+1\right)}{\Gamma\left(\frac{n+2}{p}+1\right)} M_{2,2}} \\
& =\frac{n_{1} n_{2}}{n^{2}} \cdot \frac{1}{\alpha_{1}^{n_{1}} \alpha_{2}^{n_{2}}} \cdot \frac{\Gamma\left(\frac{n+2}{p}+1\right)^{2}}{\Gamma\left(\frac{n+4}{p}+1\right)} \cdot \frac{1}{\Gamma\left(\frac{n_{1}}{p}+1\right) \Gamma\left(\frac{n_{2}}{p}+1\right)} \\
& =\frac{n_{1} n_{2}}{n^{2}} \cdot \frac{1}{g(n, 1 / p)} \cdot
\end{aligned}
$$

Summing up, we obtain

$$
\begin{aligned}
& \text { (1.8) } \frac{M_{4, n}}{M_{2, n}^{2}}\left(K_{1} *_{p} K_{2}\right) \\
& =\frac{1}{n^{2} g(n, 1 / p)}\left(n_{1}^{2} g\left(n_{1}, \frac{1}{p}\right) \frac{M_{4, n_{1}}}{M_{2, n_{1}}^{2}}\left(K_{1}\right)+2 n_{1} n_{2}+n_{2}^{2} g\left(n_{2}, \frac{1}{p}\right) \frac{M_{4, n_{2}}}{M_{2, n_{2}}^{2}}\left(K_{2}\right)\right) .
\end{aligned}
$$

\section{Permanence of inequalities for the second and fourth mo-} ments. We assume that $n_{j}, K_{j}$ are as in Section 1 , for $j=1,2$.

TheOREM 2.1. The function $p \mapsto M_{2, n}\left(K_{1} *_{p} K_{2}\right)$ is strictly decreasing on $[1,2]$, strictly increasing on $[2, \infty]$; in particular, it attains its minimum for $p=2$. Moreover, $M_{2, n}\left(K_{1} *_{p} K_{2}\right)$ attains its maximum for $p=\infty$. If $K_{1}$ and $K_{2}$ satisfy (0.1), then $K_{1} *_{p} K_{2}$ also satisfies (0.1) for all $p \in[1, \infty]$.

Proof. The function $f\left(n_{1}, 1 / p\right) f\left(n_{2}, 1 / p\right) / f(n, 1 / p)$ occurring in the expression for $M_{2, n}\left(K_{1} *_{p} K_{2}\right)$, see (1.5), is treated by computing the logarithmic derivative

$$
\begin{aligned}
& \partial_{\alpha} \ln \frac{f\left(n_{1}, \alpha\right) f\left(n_{2}, \alpha\right)}{f(n, \alpha)} \\
& \quad=\partial_{\alpha} \ln f(n, \alpha)\left(\frac{\partial_{\alpha} \ln f\left(n_{1}, \alpha\right)}{\partial_{\alpha} \ln f(n, \alpha)}+\frac{\partial_{\alpha} \ln f\left(n_{2}, \alpha\right)}{\partial_{\alpha} \ln f(n, \alpha)}-1\right) .
\end{aligned}
$$

By Corollary 4.3 below, the second factor is strictly increasing and vanishes at $\alpha=1 / 2$, whereas the first factor is positive. This implies the first assertion.

It is also clear that $M_{2, n}\left(K_{1} *_{p} K_{2}\right)$ attains its maximum for $p=1$ or $p=\infty$. In order to exclude $p=1$ it is therefore sufficient to show

$$
\frac{f\left(n_{1}, 1\right) f\left(n_{2}, 1\right)}{f(n, 1)} \leqslant 1 \quad\left(=\frac{f\left(n_{1}, 0\right) f\left(n_{2}, 0\right)}{f(n, 0)}\right) .
$$

This is shown subsequently in Lemma 2.2. 
In order to prove the last statement it is therefore sufficient to check (0.1) for $p=\infty$, i.e., the isotropic normalized cartesian product. This case, however, has been treated in [9].

Lemma 2.2. (a) The function

$$
k \mapsto \frac{f(k-1,1)}{f(k, 1)},
$$

where

$$
f(k, 1)=\frac{\Gamma((k+2)+1)^{k}}{\Gamma(k+1)^{k+2}}=\frac{(k+2) !^{k}}{k !^{k+2}}=\frac{((k+1)(k+2))^{k}}{k !^{2}}
$$

(see (1.6)), is decreasing in $k \in \mathbb{N}$.

(b) If $n, n_{1}, n_{2} \in \mathbb{N}_{0}, n=n_{1}+n_{2}$, then

$$
\frac{f\left(n_{1}, 1\right) f\left(n_{2}, 1\right)}{f(n, 1)} \leqslant 1 .
$$

Proof. (a) An elementary computation yields

$$
\frac{f(k-1,1)}{f(k, 1)}=\frac{k}{k+1}\left(\frac{k}{k+2}\right)^{k}=\frac{k}{k+1}\left(1-\frac{2}{k+2}\right)^{k} .
$$

For $k=1,2$ we obtain

$$
\frac{f(0,1)}{f(1,1)}=\frac{f(1,1)}{f(2,1)}=\frac{1}{6} .
$$

For the logarithmic derivative we estimate

$$
\begin{aligned}
\frac{d}{d k} \ln \left(\frac{k}{k+1}\right. & \left.\left(1-\frac{2}{k+2}\right)^{k}\right) \\
& =\frac{1}{k}-\frac{1}{k+1}+\ln \left(1-\frac{2}{k+2}\right)+\frac{k}{k}-\frac{k}{k+2} \\
& \leqslant \frac{1}{k}-\frac{1}{k+1}+\frac{2}{k+2}-\left(\frac{2}{k+2}+\frac{2}{(k+2)^{2}}+\frac{8}{3(k+2)^{3}}\right) \\
& =\frac{-k^{3}-\frac{8}{3} k^{2}+\frac{16}{3} k+8}{k(k+1)(k+2)^{3}}
\end{aligned}
$$

and this is $\leqslant 0$ for $k \geqslant 2$.

(b) For arbitrary $n \in \mathbb{N}_{0}$ we obviously have $f(0,1) f(n, 1) / f(n, 1)=1$. If $n_{1}, n_{2} \in \mathbb{N}_{0}, n_{1}<n_{2}, n_{1}+n_{2}=n$, then (a) implies

$$
\frac{f\left(n_{1}+1,1\right) f\left(n_{2}-1,1\right)}{f(n, 1)} \leqslant \frac{f\left(n_{1}, 1\right) f\left(n_{2}, 1\right)}{f(n, 1)},
$$

which by induction is $\leqslant 1$. 
Theorem 2.3. There exists $p_{0} \in[2, \infty)$ such that

$$
p \mapsto \frac{M_{4, n}}{M_{2, n}^{2}}\left(K_{1} *_{p} K_{2}\right)
$$

is strictly decreasing on $\left[1, p_{0}\right]$ and strictly increasing on $\left[p_{0}, \infty\right]$. Here $p_{0}=2$ if and only if $K_{j}=B_{n_{j}, 2}$, the normalized Euclidean ball in $\mathbb{R}^{n_{j}}$, for $j=1,2$. In particular, $\left(M_{4, n} / M_{2, n}^{2}\right)\left(K_{1} *_{p} K_{2}\right)$ attains its maximum for $p=1$ or $p=\infty$. If $K_{1}$ and $K_{2}$ satisfy $(0.2)$, then $K_{1} *_{p} K_{2}$ also satisfies (0.2) for all $p \in[1, \infty]$.

As a preparation for the proof, we treat the expression occurring in (1.8) in a more general setting.

LEMmA 2.4. Let $n, n_{1}, n_{2}>0, n>n_{1}, n_{2}, c_{1}, d>0, c_{2} \geqslant 0$, and define

$$
G(\alpha):=\frac{1}{g(n, \alpha)}\left(c_{1} g\left(n_{1}, \alpha\right)+d+c_{2} g\left(n_{2}, \alpha\right)\right)
$$

for $\alpha \geqslant 0$. Then there exists $\alpha_{0}>0$ such that $G$ is strictly decreasing on $\left[0, \alpha_{0}\right]$ and strictly increasing on $\left[\alpha_{0}, \infty\right)$. In particular, $G$ attains its maximum on $[0,1]$ at $\alpha=0$ or at $\alpha=1$.

Proof. We compute

$$
\begin{aligned}
G^{\prime}(\alpha)= & \frac{1}{g(n, \alpha)}\left(c_{1} \partial_{\alpha} g\left(n_{1}, \alpha\right)+c_{2} \partial_{\alpha} g\left(n_{2}, \alpha\right)\right) \\
& -\frac{\partial_{\alpha} g(n, \alpha)}{g(n, \alpha)^{2}}\left(c_{1} g\left(n_{1}, \alpha\right)+d+c_{2} g\left(n_{2}, \alpha\right)\right) \\
= & \frac{\partial_{\alpha} \ln g(n, \alpha)}{g(n, \alpha)}\left[c_{1}\left(\frac{\partial_{\alpha} \ln g\left(n_{1}, \alpha\right)}{\partial_{\alpha} \ln g(n, \alpha)}-1\right) g\left(n_{1}, \alpha\right)-d\right. \\
& \left.+c_{2}\left(\frac{\partial_{\alpha} \ln g\left(n_{2}, \alpha\right)}{\partial_{\alpha} \ln g(n, \alpha)}-1\right) g\left(n_{2}, \alpha\right)\right] .
\end{aligned}
$$

By Corollary 4.3, the second factor is strictly increasing (note that $g(n, \alpha)$ is increasing, by the positivity of $\partial_{\alpha} \ln g(n, \alpha)$ ) and negative for small $\alpha$ since $d>0$. Since the first factor is positive it follows that $G^{\prime}(\alpha)$ has a unique zero $\alpha_{0}$. (It is not difficult to show that, as $\alpha \rightarrow \infty$, the second factor tends to infinity because $g\left(n_{1}, \alpha\right) \rightarrow \infty$.)

Proof of Theorem 2.3. Because of formula (1.8), the first statement follows from Lemma 2.4, except for the fact that $p_{0} \geqslant 2$. If $K_{j}=B_{n_{j}, 2}$ for $j=1,2$, then Theorem 5.1 below implies $p_{0}=2$; in particular, in Lemma 2.4, with $c_{j}=n_{j}^{2}\left(M_{4, n_{j}} / M_{2, n_{j}}^{2}\right)\left(B_{n_{j}, 2}\right)(j=1,2), d=2 n_{1} n_{2}$, one has $G^{\prime}(1 / 2)=0$. Now, Theorem 5.1 implies $G^{\prime}(1 / 2)>0$ in all other cases (cf. formula (2.1)), i.e., $p_{0}>2$. 
In order to prove the last statement it is therefore sufficient to check $(0.2)$ for $p=1$ and $p=\infty$, i.e., the isotropic normalized join and the isotropic normalized cartesian product. These cases have been treated in [9].

3. Second and fourth moments for the $p$-balls. For $n \in \mathbb{N}$, $p \in[1, \infty]$, we denote by $B_{n, p}$ the normalized ball in $\mathbb{R}^{n}$ corresponding to the $p$-norm $|\cdot|_{p}$. The volume of the unit $p$-ball

$$
\check{B}_{n, p}:=\left\{x \in \mathbb{R}^{n}:|x|_{p} \leqslant 1\right\}
$$

is given by

$$
\operatorname{vol}_{n}\left(\check{B}_{n, p}\right)=\frac{(2 \Gamma(1 / p+1))^{n}}{\Gamma(n / p+1)}
$$

(cf. [7, p. 11]); therefore

$$
B_{n, p}=\frac{\Gamma(n / p+1)^{1 / n}}{2 \Gamma(1 / p+1)} \check{B}_{n, p} .
$$

For $n_{1}, n_{2} \in \mathbb{N}, n=n_{1}+n_{2}$, one obviously obtains

$$
B_{n, p}=B_{n_{1}, p} *_{p} B_{n_{2}, p},
$$

and therefore recursion formulas for $M_{2, n}\left(B_{n, p}\right)$ and $\left(M_{4, n} / M_{2, n}^{2}\right)\left(B_{n, p}\right)$ are obtained from (1.5) and (1.8).

Taking into account

$$
M_{2,1}\left(B_{1, p}\right)=\frac{1}{12}, \quad \frac{M_{4,1}}{M_{2,1}^{2}}\left(B_{1, p}\right)=\frac{9}{5}
$$

one obtains

$$
\begin{aligned}
& M_{2, n}\left(B_{n, p}\right)=\frac{n}{12} \cdot \frac{f(1,1 / p)}{f(n, 1 / p)^{1 / n}} \\
& \frac{M_{4, n}}{M_{2, n}^{2}}\left(B_{n, p}\right)=\frac{1}{g(n, 1 / p)}\left(\frac{9}{5 n} g\left(1, \frac{1}{p}\right)+1-\frac{1}{n}\right) .
\end{aligned}
$$

THEOREM 3.1. The functions

$$
p \mapsto M_{2, n}\left(B_{n, p}\right), \quad p \mapsto \frac{M_{4, n}}{M_{2, n}^{2}}\left(B_{n, p}\right)
$$

are strictly decreasing on $[1,2]$ and strictly increasing on $[2, \infty]$.

Proof. For the first function we compute the logarithmic derivative

$$
\partial_{\alpha} \ln \frac{f(1, \alpha)}{f(n, \alpha)^{1 / n}}=\partial_{\alpha} \ln f(n, \alpha)\left(\frac{\partial_{\alpha} \ln f(1, \alpha)}{\partial_{\alpha} \ln f(n, \alpha)}-\frac{1}{n}\right) .
$$

By Corollary 4.3 below, this implies the assertion for the first function as in the proof of Theorem 2.1. 
For the second function, we apply Lemma 2.4 (with $c_{2}=0$ ); note that $\alpha_{0}=1 / 2$ follows from Theorem 5.1 below (or can be obtained by direct computation).

4. Monotonicity properties of the $\Gamma$-function. In this section we study properties of functions of the type

$$
F(\alpha):=\prod_{j=1}^{N} \Gamma\left(\alpha n_{j}+1\right)^{\gamma_{j}} \quad(\alpha \geqslant 0),
$$

where $n_{j} \geqslant 0, \gamma_{j} \in \mathbb{R}(j=1, \ldots, N)$. We will make use of the following integral representation of the logarithmic derivative of the $\Gamma$-function:

$$
\Psi(x):=\frac{d}{d x} \ln \Gamma(x+1)=\int_{0}^{\infty}\left(\frac{e^{-t}}{t}-\frac{e^{-x t}}{e^{t}-1}\right) d t
$$

(cf. [5, $\S 1$, formula (60)] or $[8,6.351]$ ). If $\sum_{j=1}^{N} \gamma_{j} n_{j}=0$ then the logarithmic derivative of $F$ can be expressed as

$$
\begin{aligned}
\frac{d}{d \alpha} \ln F(\alpha) & =\sum_{j} \gamma_{j} n_{j} \Psi\left(\alpha n_{j}\right) \\
& =-\int_{0}^{\infty} \frac{1}{e^{t}-1} \sum_{j} \gamma_{j} n_{j} e^{-\alpha n_{j} t} d t \\
& =\frac{1}{4 \alpha} \int_{0}^{\infty} \frac{1}{\sinh ^{2}(t / 2)} \sum_{j} \gamma_{j}\left(e^{-\alpha n_{j} t}-1\right) d t
\end{aligned}
$$

where in the last step we used partial integration and

$$
\frac{d}{d t}\left(\frac{1}{e^{t}-1}\right)=-\frac{e^{t}}{\left(e^{t}-1\right)^{2}}=-\frac{1}{4 \sinh ^{2}(t / 2)} .
$$

Note that the functions $f$ and $g$ needed in Sections 2 and 3 are of the above type, with $\sum_{j} \gamma_{j} n_{j}=0$.

Proposition 4.1. Let $k_{j} \geqslant 0, m_{j} \in \mathbb{R}(j=1, \ldots, N)$ be such that the function

$$
\eta(x):=\sum_{j} m_{j} x^{k_{j}}
$$

has no zeros on $(0,1)$.

(a) Define $\varphi(n, \alpha):=\prod_{j} \Gamma\left(\alpha\left(n+k_{j}\right)+1\right)^{m_{j} /\left(n+k_{j}\right)}(n>0, \alpha \geqslant 0)$ and assume $\eta(1)=0$. Then

$$
\partial_{\alpha} \ln \varphi(n, \alpha)=-\frac{1}{\alpha} \int_{0}^{\infty} \frac{\eta\left(e^{-t}\right)}{e^{t / \alpha}-1} e^{-n t} d t \quad(\alpha>0)
$$


and, for $n>n_{1}>0$, the function

$$
Q(\alpha):=\frac{\partial_{\alpha} \ln \varphi\left(n_{1}, \alpha\right)}{\partial_{\alpha} \ln \varphi(n, \alpha)}
$$

is strictly increasing on $(0, \infty)$. If , in addition, $\eta^{\prime}(1) \neq 0$ then $Q(\alpha) \rightarrow 1$ as $\alpha \rightarrow 0$.

(b) Define $\psi(n, \alpha):=\prod_{j} \Gamma\left(\alpha\left(n+k_{j}\right)+1\right)^{m_{j}}(n, \alpha \geqslant 0)$ and assume $\eta(1)=\eta^{\prime}(1)=0$. Then

$$
\partial_{\alpha} \ln \psi(n, \alpha)=\frac{1}{4 \alpha^{2}} \int_{0}^{\infty} \frac{\eta\left(e^{-t}\right)}{\sinh ^{2}(t /(2 \alpha))} e^{-n t} d t \quad(\alpha>0)
$$

and, for $n>n_{1} \geqslant 0$, the function

$$
Q(\alpha):=\frac{\partial_{\alpha} \ln \psi\left(n_{1}, \alpha\right)}{\partial_{\alpha} \ln \psi(n, \alpha)}
$$

is strictly increasing on $(0, \infty)$. If , in addition, $\eta^{\prime \prime}(1) \neq 0$ then $Q(\alpha) \rightarrow 1$ as $\alpha \rightarrow 0$.

For the proof we need the following elementary tool.

Lemma 4.2. Let $f_{1}, f_{2}, h:(0, \infty) \rightarrow(0, \infty)$ be continuous functions, $f_{2} / f_{1}$ strictly increasing, and $h$ strictly increasing. Then

$$
\frac{\int_{0}^{\infty} f_{1}(t) h(t) d t}{\int_{0}^{\infty} f_{1}(t) d t}<\frac{\int_{0}^{\infty} f_{2}(t) h(t) d t}{\int_{0}^{\infty} f_{2}(t) d t} .
$$

(All the integrals are assumed to exist.)

Proof. Without restriction $\int_{0}^{\infty} f_{1}(t) d t=\int_{0}^{\infty} f_{2}(t) d t=1$. Then

$$
\int_{0}^{\infty}\left(\frac{f_{2}}{f_{1}}-1\right) f_{1} d t=0
$$

so there exists $\tau>0$ such that $f_{2}(\tau) / f_{1}(\tau)=1$. We conclude

$$
\begin{aligned}
\int_{0}^{\infty}\left(f_{2}-f_{1}\right) h d t & =\int_{0}^{\tau}\left(\frac{f_{2}}{f_{1}}-1\right) f_{1} h d t+\int_{\tau}^{\infty}\left(\frac{f_{2}}{f_{1}}-1\right) f_{1} h d t \\
& >h(\tau) \int_{0}^{\infty}\left(\frac{f_{2}}{f_{1}}-1\right) f_{1} d t=0 .
\end{aligned}
$$

Proof of Proposition 4.1. Without restriction assume $\eta \geqslant 0$. (Otherwise replace $m_{j}$ by $-m_{j}$ for $j=1, \ldots, N$.)

(a) Since $\sum_{j}\left(m_{j} /\left(n+k_{j}\right)\right)\left(n+k_{j}\right)=\eta(1)=0$ we obtain from (4.1) the formula 


$$
\begin{aligned}
\partial_{\alpha} \ln \varphi(n, \alpha) & =-\int_{0}^{\infty} \frac{1}{e^{t}-1} e^{-\alpha n t} \eta\left(e^{-\alpha t}\right) d t \\
& =-\frac{1}{\alpha} \int_{0}^{\infty} \frac{\eta\left(e^{-t}\right)}{e^{t / \alpha}-1} e^{-n t} d t .
\end{aligned}
$$

Letting

$$
f_{\alpha}(t):=\frac{\eta\left(e^{-t}\right)}{e^{t / \alpha}-1} e^{-n t} \quad \text { and } \quad h(t):=e^{\left(n-n_{1}\right) t}
$$

we get

$$
Q(\alpha)=\frac{\int_{0}^{\infty} f_{\alpha}(t) h(t) d t}{\int_{0}^{\infty} f_{\alpha}(t) d t}
$$

By Lemma 4.2, for the proof of the strict monotonicity of $Q$ it remains to show that, for $0<\alpha_{1}<\alpha_{2}$, the function

$$
\frac{f_{\alpha_{2}}(t)}{f_{\alpha_{1}}(t)}=\frac{e^{t / \alpha_{1}}-1}{e^{t / \alpha_{2}}-1}
$$

is strictly increasing (on $(0, \infty))$, or equivalently, that $s \mapsto\left(e^{p s}-1\right) /\left(e^{s}-1\right)$, where $p>1$, is strictly increasing. The numerator of the derivative of this function,

$$
\frac{p-1}{p} e^{(p+1) s}+\frac{1}{p} e^{s}-e^{p s},
$$

is positive by the convexity of the exponential function.

For the proof of $Q(\alpha) \rightarrow 1(\alpha \rightarrow 0)$ we note that

$$
\partial_{\alpha} \ln \varphi(n, 0)=0, \quad \partial_{\alpha}^{2} \ln \varphi(n, 0)=\int_{0}^{\infty} \frac{t}{e^{t}-1} \eta^{\prime}(1) d t
$$

by (4.2) and the assumption $\eta(1)=0$, so we are done by de l'Hospital's rule.

(b) Since $\sum_{j} m_{j}=\eta(1)=0$ and $\sum_{j} m_{j}\left(n+k_{j}\right)=n \eta(1)+\eta^{\prime}(1)=0$ we obtain from (4.1) the formula

$$
\begin{aligned}
\partial_{\alpha} \ln \psi(n, \alpha) & =\frac{1}{4 \alpha} \int_{0}^{\infty} \frac{1}{\sinh ^{2}(t / 2)} e^{-\alpha n t} \eta\left(e^{-\alpha t}\right) d t \\
& =\frac{1}{4 \alpha^{2}} \int_{0}^{\infty} \frac{\eta\left(e^{-t}\right)}{\sinh ^{2}(t /(2 \alpha))} e^{-n t} d t .
\end{aligned}
$$

Letting

$$
f_{\alpha}(t):=\frac{\eta\left(e^{-t}\right)}{\sinh ^{2}(t /(2 \alpha))} e^{-n t} \quad \text { and } \quad h(t):=e^{\left(n-n_{1}\right) t},
$$

we get

$$
Q(\alpha)=\frac{\int_{0}^{\infty} f_{\alpha}(t) h(t) d t}{\int_{0}^{\infty} f_{\alpha}(t) d t}
$$


Again by Lemma 4.2, for the proof of the strict monotonicity of $Q$ it remains to show that, for $0<\alpha_{1}<\alpha_{2}$, the function

$$
\frac{f_{\alpha_{2}}(t)}{f_{\alpha_{1}}(t)}=\left(\frac{\sinh \left(t /\left(2 \alpha_{1}\right)\right)}{\sinh \left(t /\left(2 \alpha_{2}\right)\right)}\right)^{2}
$$

is strictly increasing (on $(0, \infty)$ ), or equivalently, that $s \mapsto \sinh (p s) / \sinh s$, where $p>1$, is strictly increasing. Differentiation transforms this into the question whether

$$
p \operatorname{coth}(p s)>\operatorname{coth} s \text {. }
$$

The latter is a consequence of the strict monotonicity of $(0, \infty) \ni p \mapsto$ $p \operatorname{coth} p$, which in turn follows from

$$
\frac{d}{d p}(p \operatorname{coth} p)=\frac{\cosh p \sinh p-p}{(\sinh p)^{2}}>\frac{1 \cdot p-p}{(\sinh p)^{2}}=0 .
$$

For the proof of $Q(\alpha) \rightarrow 1(\alpha \rightarrow 0)$ we apply de l'Hospital's rule twice to the first representation of $\partial_{\alpha} \ln \psi(n, \alpha)$ in (4.3):

$$
\lim _{\alpha \rightarrow 0} Q(\alpha)=\lim _{\alpha \rightarrow 0} \frac{\alpha \partial_{\alpha} \ln \psi\left(n_{1}, \alpha\right)}{\alpha \partial_{\alpha} \ln \psi(n, \alpha)}=\lim _{\alpha \rightarrow 0} \frac{\partial_{\alpha}^{2}\left(\alpha \partial_{\alpha} \ln \psi\left(n_{1}, \alpha\right)\right)}{\partial_{\alpha}^{2}\left(\alpha \partial_{\alpha} \ln \psi(n, \alpha)\right)}=1
$$

since

$$
\left.\partial_{\alpha}^{2}\left(\alpha \partial_{\alpha} \ln \psi(n, \alpha)\right)\right|_{\alpha=0}=\frac{1}{4} \int_{0}^{\infty} \frac{t^{2}}{\sinh ^{2}(t / 2)} \eta^{\prime \prime}(1) d t \quad \text { for all } n \geqslant 0,
$$

by the assumption $\eta(1)=\eta^{\prime}(1)=0$.

In the following corollary we apply Proposition 4.1 to the functions $f$ and $g$ used in Sections 2 and 3.

Corollary 4.3. As in (1.6) and (1.7), let

$$
\begin{aligned}
f(n, \alpha) & :=\frac{\Gamma(\alpha(n+2)+1)^{n}}{\Gamma(\alpha n+1)^{n+2}}, \\
g(n, \alpha) & :=\frac{\Gamma(\alpha n+1) \Gamma(\alpha(n+4)+1)}{\Gamma(\alpha(n+2)+1)^{2}} \quad(n, \alpha \geqslant 0) .
\end{aligned}
$$

Then, for $n>n_{1}>0$, the functions

$$
\alpha \mapsto \frac{\partial_{\alpha} \ln f\left(n_{1}, \alpha\right)}{\partial_{\alpha} \ln f(n, \alpha)} \quad \text { and } \quad \alpha \mapsto \frac{\partial_{\alpha} \ln g\left(n_{1}, \alpha\right)}{\partial_{\alpha} \ln g(n, \alpha)}
$$

are strictly increasing, $\partial_{\alpha} \ln f$ and $\partial_{\alpha} \ln g$ are positive on $(0, \infty) \times(0, \infty)$, $\partial_{\alpha} \ln f(n, 1 / 2)=2 n$, and

$$
\frac{\partial_{\alpha} \ln g\left(n_{1}, \alpha\right)}{\partial_{\alpha} \ln g(n, \alpha)} \rightarrow 1 \quad \text { as } \alpha \rightarrow 0 .
$$

Proof. To prove the statements about $f$ we define $\varphi:=f^{1 /(n(n+2))}$. Then $\varphi$ is of the type considered in Proposition 4.1(a), with $k_{1}=2, k_{2}=0$, 
$m_{1}=1, m_{2}=-1$. The corresponding function $\eta$ is $\eta(x)=x^{2}-1$, so it satisfies the conditions of Proposition 4.1(a). Hence

$$
\frac{\partial_{\alpha} \ln f\left(n_{1}, \alpha\right)}{\partial_{\alpha} \ln f(n, \alpha)}=\frac{n_{1}\left(n_{1}+2\right)}{n(n+2)} \cdot \frac{\partial_{\alpha} \ln \varphi\left(n_{1}, \alpha\right)}{\partial_{\alpha} \ln \varphi(n, \alpha)}
$$

is strictly increasing and

$$
\partial_{\alpha} \ln \varphi(n, \alpha)=\frac{1}{\alpha} \int_{0}^{\infty} \frac{1-e^{-2 t}}{e^{t / \alpha}-1} e^{-n t} d t>0 \quad(\alpha>0) .
$$

For $\alpha=1 / 2$ we obtain

$$
\partial_{\alpha} \ln f(n, 1 / 2)=n(n+2) \partial_{\alpha} \ln \varphi(n, 1 / 2)=2 n(n+2) \int_{0}^{\infty} e^{-(n+2) t} d t=2 n .
$$

The function $g$ is of the type considered in Proposition 4.1(b), with $k_{1}=0, k_{2}=4, k_{3}=2, m_{1}=m_{2}=1, m_{3}=-2$. The corresponding function $\eta$ is $\eta(x)=1+x^{4}-2 x^{2}=\left(1-x^{2}\right)^{2} \geqslant 0$, so an application of Proposition 4.1(b) yields the first statement and the last statement about $g$. The positivity of $\partial_{\alpha} \ln g$ follows from (4.3).

5. The minimum of $M_{4, n} M_{0, n} / M_{2, n}^{2}$. The result of this section will be formulated for the more general context of compact subsets of $\mathbb{R}^{n}$ of non-vanishing volume which are star-shaped with respect to the origin. A body $K$ like this is described by its radial function $\varrho=\varrho_{K}: S^{n-1} \rightarrow[0, \infty)$,

$$
\varrho(u):=\max \{r \geqslant 0: r u \in K\} .
$$

The moments of $K$ are then given by

$$
\begin{aligned}
& M_{0, n}(K)=\operatorname{vol}(K)=\frac{1}{n} \int_{S^{n-1}} \varrho(u)^{n} d S(u), \\
& M_{2, n}(K)=\frac{1}{n+2} \int_{S^{n-1}} \varrho(u)^{n+2} d S(u), \\
& M_{4, n}(K)=\frac{1}{n+4} \int_{S^{n-1}} \varrho(u)^{n+4} d S(u) .
\end{aligned}
$$

TheOREM 5.1. Let $K \subseteq \mathbb{R}^{n}$ be compact and star-shaped with respect to the origin, with the origin contained in the interior of $K$. Then

$$
\frac{M_{4, n} M_{0, n}}{M_{2, n}^{2}}(K) \geqslant \frac{M_{4, n} M_{0, n}}{M_{2, n}^{2}}\left(B_{n, 2}\right),
$$

with equality if and only if $K$ is a multiple of the Euclidean ball $B_{n, 2}$.

Proof. In contrast to the remainder of this paper, we shall choose the scaling of $K$ in such a way that the second moment is normalized to be 
$M_{2, n}(K)=n$. By $\varrho_{0}$ we denote the radius of the accordingly scaled Euclidean ball,

$$
\varrho_{0}=\left(\frac{n(n+2)}{\sigma_{n-1}}\right)^{1 /(n+2)}=\left(\frac{n+2}{\omega_{n}}\right)^{1 /(n+2)} .
$$

Denoting by $\mu_{n-1}$ the surface measure on $S^{n-1}$, normalized to be a probability measure, we then have to show

$$
\int \varrho_{0}^{n+4} d \mu_{n-1} \int \varrho_{0}^{n} d \mu_{n-1} \leqslant \int \varrho(u)^{n+4} d \mu_{n-1} \int \varrho(u)^{n} d \mu_{n-1},
$$

or equivalently,

$$
1 \leqslant \int\left(\left(\frac{\varrho}{\varrho_{0}}\right)^{n+2}\right)^{(n+4) /(n+2)} d \mu_{n-1} \int\left(\left(\frac{\varrho}{\varrho_{0}}\right)^{n+2}\right)^{n /(n+2)} d \mu_{n-1},
$$

with equality if and only if $\varrho=\varrho_{0}$. Note that for $p:=(n+4) /(n+2)$, $q:=n /(n+2)$ one has $0<q<p<2, p+q=2$, and that $f:=\left(\varrho / \varrho_{0}\right)^{n+2}$ is strictly positive on $S^{n-1}$, with $\int f d \mu_{n-1}=1$. Therefore the conclusion is a consequence of the Cauchy-Schwarz inequality,

$$
1=\int f^{p / 2+q / 2} d \mu_{n-1} \leqslant\left(\int f^{p} d \mu_{n-1}\right)^{1 / 2}\left(\int f^{q} d \mu_{n-1}\right)^{1 / 2},
$$

where equality holds if and only if $f^{p / 2}=c f^{q / 2}$ for some constant $c$. Because of $p \neq q$, the latter is equivalent to $f=1$.

Remark 5.2. For any compact set $K$ in $\mathbb{R}^{n}$, one has

$$
\left(\int_{K}|x|^{2} d x\right)^{2} \leqslant \int_{K}|x|^{4} d x \int_{K} d x
$$

by the Cauchy-Schwarz inequality. Theorem 5.1 tells us that, for compact sets $K \subseteq \mathbb{R}^{n}$ which are star-shaped with respect to the origin, this inequality can be improved to

with

$$
\left(\int_{K}|x|^{2} d x\right)^{2} \leqslant c_{0} \int_{K}|x|^{4} d x \int_{K} d x
$$

$$
c_{0}=\frac{n^{2}}{\left(\sigma_{n-1} /(n+4)\right) \varrho_{0}^{n+4}\left(\sigma_{n-1} / n\right) \varrho_{0}^{n}}=\frac{n(n+4)}{(n+2)^{2}}<1 .
$$

This conclusion makes it clear that the inequality of Theorem 5.1 is not true for arbitrary compact subsets of $\mathbb{R}^{n}$. (Take, e.g., thin spherical shells!)

\section{References}

[1] M. Anttila, K. Ball and I. Perissinaki, The central limit problem for convex bodies, preprint, 1998.

[2] U. Brehm, P. Hinow, H. Vogt and J. Voigt, Moment inequalities and central limit properties of isotropic convex bodies, Math. Z., to appear. 
[3] U. Brehm and J. Voigt, Asymptotics of cross sections for convex bodies, Beiträge Algebra Geom. 41 (2000), 437-454.

[4] A. Koldobsky and M. Lifshits, Average volume of sections of star bodies, in: Geometric Aspects of Functional Analysis, V. D. Milman and G. Schechtman (eds.), Lecture Notes in Math. 1745, Springer, Berlin, 2000, 119-146.

[5] A. Kratzer and W. Franz, Transzendente Funktionen, Akad. Verlagsanstalt Geest \& Portig, Leipzig, 1960.

[6] V. D. Milman and A. Pajor, Isotropic position and inertia ellipsoids and zonoids of the unit ball of a normed n-dimensional space, in: Geometric Aspects of Functional Analysis, J. Lindenstrauss and V. D. Milman (eds.), Lecture Notes in Math. 1376, Springer, Berlin, 1989, 64-104.

[7] G. Pisier, The Volume of Convex Bodies and Banach Space Geometry, Cambridge Univ. Press, Cambridge, 1989.

[8] I. M. Ryshik and I. S. Gradstein, Summen-, Produkt- und Integral-Tafeln, Deutscher Verlag Wiss., Berlin, 1957.

[9] J. Voigt, A concentration of mass property for isotropic convex bodies in high dimensions, Israel J. Math. 115 (2000), 235-251.

[10] H. von Weizsäcker, Sudakov's typical marginals, random linear functionals and a conditional central limit theorem, Probab. Theory Related Fields 107 (1997), $313-324$.

Fachrichtung Mathematik

Technische Universität Dresden

D-01062 Dresden, Germany

E-mail: brehm@math.tu-dresden.de

vogt@math.tu-dresden.de

voigt@math.tu-dresden.de

Received March 27, 2000

Revised version March 30, 2001 